\title{
Maps based on the phase resetting curve explain spike statistics of coupled neural oscillators observed in the presence of noise
}

\author{
Srisairam Achuthan ${ }^{1 *}$, Jianxia Cui ${ }^{2}$, Robert Butera ${ }^{3}$, Carmen C Canavier ${ }^{1,4}$ \\ From Nineteenth Annual Computational Neuroscience Meeting: CNS*2010 \\ San Antonio, TX, USA. 24-30 July 2010
}

Histograms of phase differences are often used to infer synchrony among coupled neurons. We use the phase resetting curve (PRC) measured under the assumption of pulsatile coupling in an isolated model and biological neurons to predict phase-locking when the neurons are reciprocally coupled via the dynamic clamp. For two coupled neurons without delays, the interval between when a neuron spikes and when it receives an input from the other neuron is the stimulus interval(ts), and the interval between when it receives an input and when it spikes next is the recovery interval (tr). The recovery interval in one neuron in any given cycle is equal to the stimulus interval in the other neuron by definition. We calculate the recovery interval in each neuron for each stimulus interval using the PRC, then plot the two tr/ts curves so that intervals that are equal by definition are on the same axes. The recovery interval on the curve for one neuron is mapped to the stimulus interval on the other curve, tracing a stair-step pattern (see Fig. 1C). For the case in which a stable intersection (Fig. 1C)

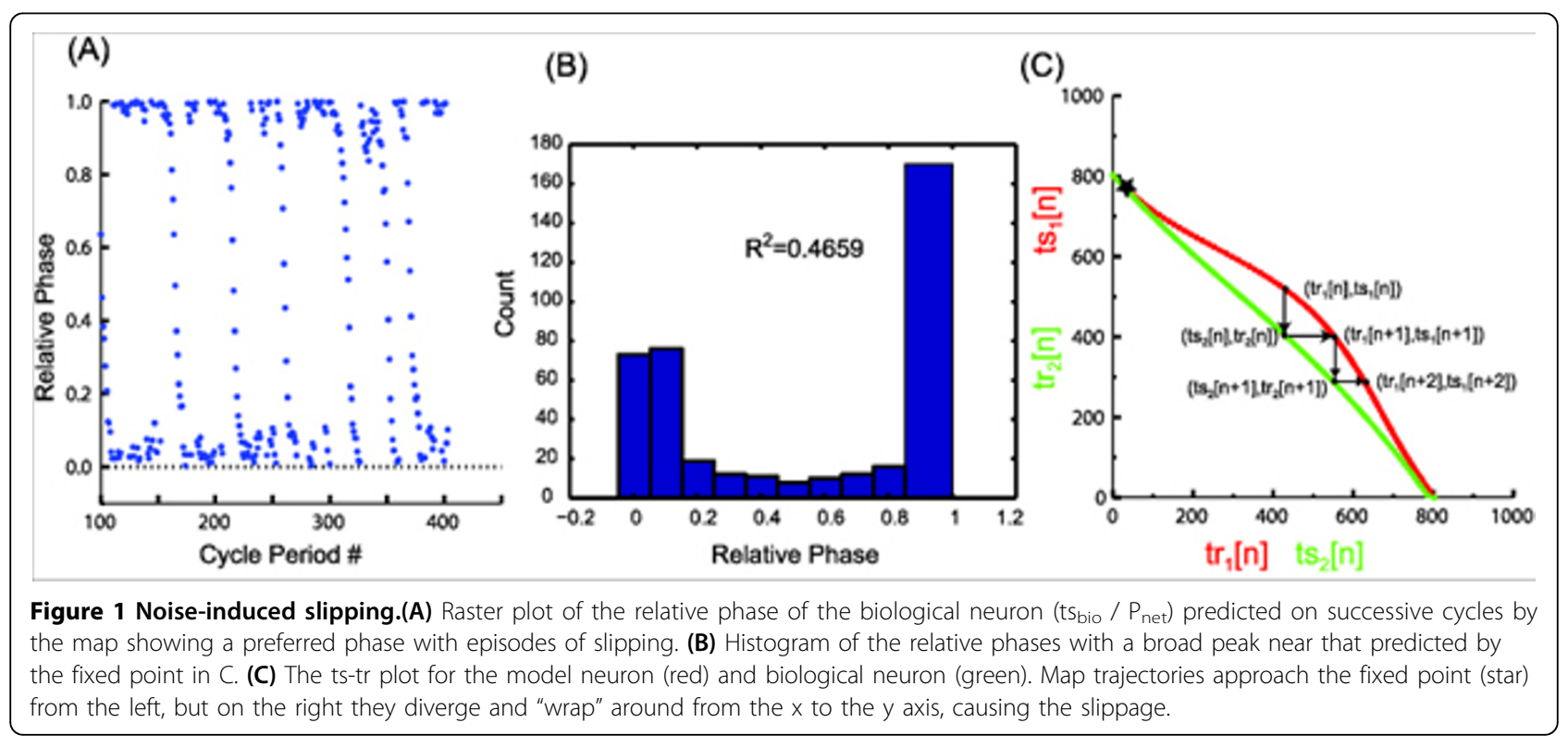

\footnotetext{
* Correspondence: sachut@lsuhsc.edu

${ }^{1}$ Neuroscience Center of Excellence, Louisiana State University Health Sciences Center, New Orleans, LA, 70112, USA
} 
predicts phase-locking without noise, the map output with Gaussian noise added to the PRC (Fig. 1A) is a good match to the data (not shown), as noise broadens the histogram and decreases the circular statistic $R^{2}$ (Fig. 1B). If the two curves are close in a range of phases, but never actually intersect, the network phase changes relatively slowly at those phases, resulting in a peak in the histogram of network phases that we call a weak, near-locking. The output of the map qualitatively also matches experimental data. For this case (not shown), the histogram and $R^{2}$ of the map output are not very sensitive to noise. Thus, PRCs can predict and help interpret the sensitivity of the network to noise. Modulation of noise levels could theoretically synchronize and desynchronize coupled neural oscillators that can lock precisely in the absence of noise, whereas weak, near-lockings are only slightly modulated by noise.

\section{Author details}

${ }^{1}$ Neuroscience Center of Excellence, Louisiana State University Health Sciences Center, New Orleans, LA, 70112, USA. ${ }^{2}$ Cellular Neuroengineering, Dept. of Bioengineering, University of California, San Diego, CA, 92093, USA. ${ }^{3}$ Laboratory for Neuroengineering, School of Electrical and Computer Engineering, Georgia Institute of Technology, Atlanta, GA, 30332, USA ${ }^{4}$ Department of Ophthalmology, LSU Health Sciences Center, New Orleans, LA, 70112, USA.

Published: 20 July 2010
Submit your next manuscript to BioMed Central and take full advantage of:

- Convenient online submission

- Thorough peer review

- No space constraints or color figure charges

- Immediate publication on acceptance

- Inclusion in PubMed, CAS, Scopus and Google Scholar

- Research which is freely available for redistribution

Submit your manuscript at www.biomedcentral.com/submit 